\title{
The view-based approach to dynamic inter-organizational workflow cooperation
}

Technical University of Vienna Information Systems Institute Distributed Systems Group

\author{
Issam Chebbi, Samir Tata and \\ Schahram Dustdar \\ Issam.Chebbi@int-evry.fr \\ Samir.Tata@int-evry.fr \\ dustdar@infosys.tuwien.ac.at
}

TUV-1841-2004-23 November 30, 2004

\begin{abstract}
This paper presents a novel approach to inter-organizational workflow cooperation. Our goal is to provide support for organizations which are involved in a shared but not pre-modeled cooperative workflow across organizational boundaries. Our approach allows for partial visibility of workflows and their resources, thus providing powerful ways for interorganizational workflow configuration. Varying degrees of visibility of workflows enable organizations to retain required levels of privacy and security of internal workflows. Our presented view concept provides a high degree of flexibility for participating organizations, since internal structures of collaborative workflows may be adapted without changes in the inter-organizational workflows. Furthermore, we provide workflow participants with the freedom to change their workflows without changing their roles in the cooperation. This increases flexibility and is an important step to increase efficiency as well as reduction in costs for inter-organizational workflows. The presented approach is inspired by the Service-oriented Architecture (SOA). Accordingly, our approach consists of three steps: workflow advertisement, workflow interconnection, and workflow cooperation.
\end{abstract}

Keywords: Inter-organizational Workflows, Cooperative Workflows

Argentinierstr. 8/184-1

A-1040 Vienna, Austria

phone: +43 158801-18402

(C)2004, Distributed Systems Group, Technical University of Vienna

fax: +43 1 58801-18491

URL: http://www.infosys.tuwien.ac.at/ 


\title{
The view-based approach to dynamic inter-organizational workflow cooperation
}

\author{
Issam Chebbi ${ }^{1}$, Samir Tata ${ }^{1}$, and Schahram Dustdar ${ }^{2}$ \\ 1 Department of Computer Science, GET/INT \\ (Institut National des Télécommunications) \\ 9, Rue Charles Fourier 91011 Evry, France \\ $\{$ Issam.Chebbi, Samir. Tata\}@int-evry.fr \\ ${ }^{2}$ Vienna University of Technology \\ Argentinierstrasse 8/184-1 \\ A-1040 Wien, Austria \\ dustdar@infosys.tuwien.ac.at
}

\begin{abstract}
This paper presents a novel approach to inter-organizational workflow cooperation. Our goal is to provide support for organizations which are involved in a shared but not pre-modeled cooperative workflow across organizational boundaries. Our approach allows for partial visibility of workflows and their resources, thus providing powerful ways for inter-organizational workflow configuration. Varying degrees of visibility of workflows enable organizations to retain required levels of privacy and security of internal workflows. Our presented view concept provides a high degree of flexibility for participating organizations, since internal structures of collaborative workflows may be adapted without changes in the inter-organizational workflows. Furthermore, we provide workflow participants with the freedom to change their workflows without changing their roles in the cooperation. This increases flexibility and is an important step to increase efficiency as well as reduction in costs for inter-organizational workflows. The presented approach is inspired by the Service-oriented Architecture (SOA). Accordingly, our approach consists of three steps: workflow advertisement, workflow interconnection, and workflow cooperation.
\end{abstract}

Keywords: Inter-organizational Workflows, Cooperative Workflows.

\section{Introduction}

In context of globalization, a high competitive pressure characterizes the general situation on businesses. Competition is a dilemma many organizations face every day. It can lead to intensive re-structuring of organizational structures and processes to make production and services more efficient and less expensive. Additionally, new forms of inter-organizational collaboration between organizations may emerge. In this case organizations especially Small and Medium sized Enterprises (SMEs), cooperate to fulfill conditions of complex, often concurrent projects. 
Parallel to this evolution, organizations are increasingly utilizing processaware information systems to perform their workflows in an automated way. Based on such information systems, organizations focus on their core competencies and access other competencies through cooperation, moving towards a new form of network known as virtual organization.

There is still no agreed-upon definition of virtual organizations. Broadly speaking, a virtual organization is often defined as a temporary organization formed from strategic alliances or partnerships ("real organizations") that can be dissolved when the common business or the common project is finished. For Davidow and Malone [1], the word "virtual" comes from the idea of "potential" meaning "excellent", "high quality" or "advanced". A virtual organization is considered as the modern organization form the more advanced and the most efficient [2]. Byrne defines a virtual organization as a temporary network of independent companies, suppliers, customers, and even rivals, linked by information technology to share costs, skills and access to markets. It will have neither central offices nor organizational charts, nor hierarchies, and no vertical integration [3].

As for us, we define a virtual organization as a set of partners ("real organizations") distributed in time and in space sharing resources and competencies (similar or dissimilar) and cooperating to reach some shared objectives using information technologies. Thus, partners with complementary competencies and knowledge can be gathered to carry out projects, which are not within the range of only one organization: cooperation allows each partner to benefit from knowledge of the other partners in the virtual organization. With this intention, partner workflows are not carried out in an isolated manner, but interact during their execution, while sharing common data in a coordinated way. Coordination brings a synergy that contributes to the improvement of each partner work performances.

The rest of this paper is organized as follows. Section 2 presents the existing approaches for inter-organizational workflows. Section 3 identifies the requirements our contribution aims to meet. Section 4 proposes a three steps approach for inter-organizational workflow cooperation: workflow identification and advertisement, workflow interconnection, and workflow cooperation. All steps are discussed, and the second step is presented in more detail in Section 5. Section 6 provides a brief overview on the cooperation prototype platform we are developing. Section 7 concludes and presents our future work.

\section{Related Work}

For a number of years research on workflow management has focused on interorganizational issues and much has been achieved so far.

In [4], the author presents some forms of workflow-interoperability and focuses on capacity sharing, chained execution, subcontracting, (extended) case transfer, loosely coupled, and public-to-private architectures. A workflow is seen as an "automation of a business process, in whole or part, during which doc- 
uments, information or tasks are passed from one participant to another for action, according to a set of procedural rules" [5]).

The inter-organizational cooperation problem has also been addressed by using the notion of agreements and contracts to define the business relationships between organizations. An example is the CrossFlow approach [6,7].

Moreover, the WISE project addressed the issue process crossing the organizational boundaries and developed an architecture to model a virtual enterprise process $[8,9]$. Besides, a model to support collaborative work in virtual enterprises based on process services and contracts has been proposed in [10]. Virtual enterprises are also addressed by BPEL ( Business Process Execution Language for Web Services) [11] which provides an XML notation and semantics for specifying business process behavior based on Web services.

In the following we present a brief survey of the previously mentioned work that have been done in the field of inter-organizational workflows

\subsection{Forms of workflow interoperability}

Various forms of interoperability are defined in the literature, which we briefly summarize: capacity sharing, chained execution, subcontracting, (extended) case transfer, loosely coupled, public to private approach.

Capacity sharing : tasks are executed by external resources under the control of one workflow manager.

Chained execution : the process is divided into subsequent phases and each business partner takes care of one phase. The workflow process is split into a number of disjunctive sub processes executed by different business partners in a sequential order [12]. This form of interoperability is only useful for applications where the process is composed of sequentially ordered parts. Nevertheless, it was generalized into an approach to distributed workflow execution where parts are inter-mixed [13]. However, this last approach is static since it starts from a global centralized workflow where all activities are known a priori and assumes that for each activity there exists an assignment to a department or business unit of the enterprise.

Subcontracting : a sub-process is executed by another organization. There is one business partner, which subcontracts sub processes to other business partners.

Case transfer : each partner uses the same workflow process and cases (i.e., workflow instance) are transferred from one partner to another. If at a specific location the process is extended with additional tasks, then this form is called extended case transfer. Cases can be transferred among partners. At any time, each case resides at exactly one location [14].

Loosely coupled : each partner takes care of a specified part of the process which may be active in parallel.

Public-To-Private : a common public workflow is specified and partitioned according to the organizations involved by private refinement of the parts based on a notion of inheritance. Each partner has a copy of the workflow 
process description. The public-to-private approach consists of three steps. Firstly, the organizations involved agree on a common public workflow, which serves as a contract between these organizations. Secondly, each task of the public workflow is mapped onto one of the domains (i.e., organization). Each domain is responsible for a part of the public workflow, referred to as its public part. Thirdly, each domain can now make use of its autonomy to create a private workflow. To satisfy the correctness of the overall interorganizational workflow, however, each domain may only choose a private workflow which is a subclass of its public part [15].

Problems to be encountered on the way to workflow interoperability include mainly autonomy of local workflow processing, confidentiality that prevents complete view of local workflow [16], and especially flexibility that needs no definition of a global workflow that defines cooperation between local workflows.

In [10], the authors present an approach for process management and coordination based on synchronization points between process services. This approach provides more flexibility in order to allow partners to personalize their internal processes without affecting the cooperation.

\subsection{Contracting of workflows}

CrossFlow The CrossFlow [17] project investigates some issues which are concerned with business processes crossing organizational boundaries. A contractbased approach is used to define the business relationships between the organizations. Within this contractual basis, inter-organizational processes can be defined and performed. However, the approach does not support arbitrary public processes and no standard definition language and semantics is provided for the enforcement of contracts between two enterprises. In addition, all enterprises involved are required to use the same software for contract enforcement.

WISE The WISE [8,9] (Workflow based Internet SErvices) project aims at designing, building, and testing commercial infrastructures for developing distributed applications over the Internet. It proposed a framework to compose a virtual business process through process interfaces of several enterprises. This architecture provides means to define, enact, and monitor virtual enterprises business processes as well as to manage context aware communication among process participants. It includes an Internet workflow engine to control the business process execution, a process modeling tool to define and monitor processes, and a catalog tool to find the building blocks for the processes. A workflow engine based on the Internet is supposed to overcome the shortcoming of other workflow systems by providing workflow functionality for heterogeneous, distributed applications. WISE is platform independent. The accessibility over the Internet makes this solution scalable and open but service descriptions and the service catalog are not in line with general standards. Moreover, the centralized workflow engine inhibits dynamic selection and exchange of partners since all participants have to comply with stipulated interfaces. 


\subsection{Workflow specification languages}

To specify inter-organizational workflows, big efforts have been made during recent years and many languages have been proposed. In the following we present a very brief survey of some proposed languages.

Business Process Execution Language for Web Services The Business Process Execution Language for Web Services (BPEL4WS or BPEL for short) [11] is a language for specifying business processes behavior based on Web services and business interaction protocols. It merges and extends the WSFL concepts of $I B M$ (control structures of $W S F L$ as the sequence, parallel, and loops structures) and those of XLANG of Microsoft (instantiationcorrelation, compensation. A $B P E L$ process allows the definition of two types of business processes, abstract process and executable process. The first type defines the business protocol role and describes its public aspects. The second one, defines the logic and state of the process by providing sequence of the Web service interactions conducted at each business partner. Moreover, $B P E L$ defines a set of primitive activities, such as invoke, to invoke Web service operations. These primitive activities can be combined into more complex primitives using any of the structure activities provided such as sequence, flow, and while. However, the Business Process Execution Language doesn't support many concepts that are paramount for inter-organizational collaboration. First, it doesn't profit of the rich concepts of exiting workflow management systems as the notion of manual activities, applications, nor addresses the integration with them, since it uses Web services exclusively which represent a limit to call other types of services like XML services, databases, etc. Second, in the context of collaboration, it does not support the partners' heterogeneity. Incoming messages must be validated as well as transformed and enriched with additional data which represent a rigid constraint in the context of collaboration where partners are supposed to manipulate different kind of structures and process heterogeneous soft and hard infrastructures. Moreover, the collaboration description that consists of linking roles to ports is limited. Besides, BPEL does not cater for non Web service interactions and the notion of independent activities, everything in a $B P E L$ is a web service operation. Finally, BPEL doesn't provide yet a standard way to specify how flows in the same process send messages to each other. Indeed, it is possible and critical that flows in the same process be able to send messages to each other but there is no standard way to specify that.

WSDL [18] is an XML-based language for locating and describing Web services, and how to access them. It offers four ways of message transmissions, wherby today mostly two of them are supported: one-way and request/response messages. It includes also a set of protocol bindings like SOAP, MIME and HTTP GET/POST.

WSFL [19] is built on top of $W S D L$ and can be used to refine a $W S D L$ specification or compose workflow fragments. It supports workflows fragment 
integration with heterogeneous data structures by using XPath expressions.

XLANG [20] refines $W S D L$ service specification with behavior and allows the WSDL services composition specification. It uses the notion of blocks and supports message handling, timing and execption handling. It also supports ACID transactions and open nested transactions with compensation. But transactions are not allowed to span workflow fragments.

BPML [21] presents some similarity with $X L A N G$ and provides additional concepts like executable specifications, transactions spanning workflow fragments, and dynamic participation. It also offers a visibility mechanism for information hiding.

WSCL [22] aims at defining the minimal set of concepts necessary for the conversation specification. This minimalism makes the language simple but restricts the expressiveness of its specifications. It does not support parallel activities, and timing constraints, for example.

ebXML [23] aims at providing a framework for business to business transactions. It supports re-usable data types, interorganizational transactions, and profiles and agreements. It offers interaction primitives to support timing, security, and atomicity properties.

WPDL [24] is intended for the exchange of workflow types betwwen workflow management systems but not for inter-organizational workflow specification. It also lacks interaction support.

Despite their diversity, no single language fulfills all requirements identified for specifying inter-organizational workflows [25].

Moreover, in [26] the authors have identified a number of workflow data patterns to describe how data is defined and used in workflow systems, and validated their applicability on six workflow systems and process languages. The main results of this study are :

- individual products tend to favour either task-level approach to pass data between task instances and shared data at case level.

- limited potential for achieving external integration

- lack of support for complex data structures

- lack of fully functional capabilities for dealing with potential data mismatches

- lacks of data-based trigger support

In consequence, the absence of end-to-end process control, which is often related to the absence and/or limitation of a single overall process ownership, has led workflow research to reexamine and to find new ways for workflow composition. One of the basic obstacles is the lack of a comprehensive model for inter-organizational workflows as a basis for contracting and standardization. 


\begin{tabular}{|c|c|c|c|c|}
\hline capproach & Flexibility & Heterogeneity & Privacy & $\begin{array}{c}\text { Process } \\
\text { Preservation }\end{array}$ \\
\hline \hline Capacity sharing & - & - & - & - \\
\hline Chained execution & - & - & + & + \\
\hline Subcontracting & $+/-$ & $+/-$ & + & + \\
\hline Case Transfer & - & $+/-$ & $+/-$ & $+/$ \\
\hline Loosely Coupled & $+/-$ & $+/-$ & + & + \\
\hline Public-To-Private & $+/-$ & - & $+/-$ & $+/$ \\
\hline CrossFlow & $+/-$ & - & + & + \\
\hline WISE & $+/-$ & $+/-$ & + & + \\
\hline BPEL & - & - & + & + \\
\hline
\end{tabular}

Fig. 1. Overview of related work

\section{Requirements for inter-organizational workflows}

\subsection{Flexibility Support}

Cooperation between partners within a virtual organization is established according to needs for businesses and their competencies and roles. This leads to a dynamic character of cooperations. Indeed, the set of partners is in constant evolution. It is possible for organizations composing the virtual organization to be geographically distributed. It is also possible for organizations to join and leave the virtual organization as its state changes over time. That is, the composition of the virtual organization may be dynamic. Furthermore, its shared net behavior implies that there is some dynamic structure to the interactions between the organizations composing the virtual organization.

On the one hand, interactions can be relevant and constructive if they complement each other and guide the work toward the objective of the virtual organization. On the other hand, they can cause adverse effects if they are not well coordinated or if participants don't follow their roles in the cooperation. An action's effect can unintentionally remove the effect of an other one. The effective result of the cooperation and the desired objective are then likely to diverge.

Since there is some dynamic structure to the interactions between the organizations composing the virtual organization, we think that interactions between workflows in virtual organizations cannot be specified in advance. In [12] the author states that "there are numerous situations where the organizations participating in a shared workflow processes feel the need to specify the coordination structure explicitly". In [27] the author mentions that "in many cases, where the coordination structure and the interaction between the business partners are not specified explicitly, this is not a realistic assumption". Nevertheless, we think that since interactions are dynamic their specification is difficult (if not impossible). Our intention and contribution of this paper is to describe (without explicit specification) a set of accepted interaction scenarios rather than one 
(which is the case of existing approaches since they use a workflow to specify interactions between workflows, see [15] for example).

\subsection{Privacy Respect Principle}

On one hand, cooperation needs a certain degree of workflow inter-visibility in order to perform interactions and data exchange. On the other hand, cooperation may be employed as a cover for organizations to internalize the know-how of their partners. The question here is how to best preserve the know-how of each partner and capitalize on the accumulated experience and knowledge to allow cooperation and to improve productivity.

In order to preserve privacy and autonomy of process participants, we must reduce workflow inter-visibility to be as little as the cooperations need.

\subsection{Established Workflow Preservation}

For enabling cooperative organizations to integrate their disparate workflows it is necessary to allow them to use established workflows.

When planning projects, it's important to note that any changes to established workflows (even clear improvements) will cost money and time. Therefore, if organizations are to achieve the efficiencies and reduction in costs that the cooperation promises especially if they are SMEs, approaches for workflow cooperation must fully integrate pre-established workflows.

\subsection{Summary of related work vis-à-vis inter-organizational workflows requirements}

The last years have been seen the developement of many inter-organizational workflows approachs allowing organizations with complementary skills to cooperate and carry out works that are not with the range of only one organization. Despite their existence, these proposals are facing many problems vis-à-vis inter-organizational workflows requirements that we recall are flexibility, heterogeneity, and privacy and process preservation respect. In fact, many existing approches are not very flexible and interactions are specified in advance. Moreover, almost all solutions currently developed for the management of cooperation in the virtual organizations suppose that the homogeneity of the partners in terms of data structures, business logic, for example, which is restrictive and limits the cooperation. Besides, although some solutions provide means to preserve privacy and established workflows, inter-visibility is either very tiny and the collaborating partners act as black boxes or very open. In both cases, it is not convenient in the context of inter-organizational workflows, where we need a certain degree of workflow inter-visibility in order to perform interactions and data exchange without revealing partners know-how. In the word, the inter-visibility must be as tiny as cooperation needs. Finally, the study of the existing approaches shows that no single solution fulfills all requirements identified for the inter-organizational workflow collaboration. 


\section{Steps for inter-organizational workflow cooperation}

To meet the requirements we have presented above, we propose a novel approach to inter-organizational workflow cooperation. This is motivated by the idea that an inter-organizational workflow can be considered as a cooperation of several pre-established workflows of several organizations.

The approach is inspired by the Service-oriented Architecture (SOA). That architecture requires three fundamental operations: publish, find, and bind. Service providers publish services to a service broker. Service requesters find required services using a service broker and bind to them. Accordingly, our approach consists of three steps: workflow advertisement, workflow interconnection, and workflow cooperation. In the following we present these steps. Section 5 focuses on the second step of this approach.

\subsection{Step 1: workflow identification and advertisement}

For building an inter-organizational workflow, each organization has to advertise its offered and required activities within their workflows. Each organization identifies its partners with complementary competencies and knowledge that can be gathered to carry out projects which are not within the range of only one organization. Partner identification is based on a (semi) automated search of the new organizations and potential partners, looking for joining a virtual organization. Research will be based on the semantic description of services (i.e. workflow activities), which the organization requires, and the level of the cooperation that it wishes to establish. In other words, the profiles of the workflow activities to be interconnected. The various profiles published can be managed within an accessible registry on the Web.

Each organization does not know a priori which partners to cooperate with. The registry role provides an organization with searching and publication capabilities, which allow the organization to get partners with useful skills. In addition, it gives organizations the ability to share workflow semantic information and workflow resources. Indeed, a semantic registry is the key foundation block upon which inter-organizational workflow cooperation can be built. Registry technology enables trading partners to identify common data sets, data structures, and workflows.

\subsection{Step 2: workflow interconnection using cooperation policies}

Identified partners negotiate their roles within the virtual organization as well as the coordination of their workflows. The result of this step is a set of cooperation policies that describe especially the responsibilities and the roles played by the partners in the cooperation. For each partner, cooperation policies define the visibility levels of its workflows for its partners.

The policies will describe (without explicit specification) a set of accepted interaction scenarios rather than one (which is the case of existing approaches since they use a workflow to specify interactions between workflows, see [15] 
for example). To do so, cooperation policies are defined between some virtual activities belonging to workflows of the virtual organization. A virtual activity can be connected to one or several activities belonging to one workflow and represents the level of the visibility used to preserve privacy and its know-how as well as to allow interactions with cooperating partners. The inter-visibility is reduced to be as little as is required for the cooperation (see [28-30] for more details on cooperation policies).

Connections between virtual activities and "real" ones can be changed without changing the coordination (or control flow) between virtual activities. This allows an organization to adapt and/or change its workflow internal structure without changing its role in the cooperation. Section 5 gives examples for connection between virtual and "real" activities.

\subsection{Step 3: workflow cooperation and monitoring using trusted third party}

Trust is a very important condition to guarantee when one would support electronic cooperation between workflows in virtual organizations. Indeed, [31] quote that the underlying basis for exchange in Internet marketplaces is trust among market participants. Significant efforts have been made in this area. For example, [32] propose models of a trusted third party in electronic commerce based on certification technologies. The idea is that a trusted third party acts as a certification authority, providing validation of market participants using public key encryption technology. We call this trusted third party a contracting authority. This authority will assume the monitoring and the control of cooperation between workflows.

\subsection{Cooperation Sequence Diagram}

Figure 2 depicts a sequence diagram showing the different steps described above as well as their interactions. The diagram is composed of a set of partners who will cooperate, a registry containing the participants profiles, and a contracting authority ensuring the cooperation monitoring and control. We identify three logical blocks. In the first block, partners publish some of their activities into the registry $(\operatorname{publish}(\ldots))$. Then, to carry out a work that is not with the range of only one organization, a partner begins by searching organizations with complementary skills via the activities they published (find $(. .)$.$) . When these or-$ ganizations are found, the registry informs them that they will be partners in an inter-organizational workflow $(\operatorname{partner}(\ldots))$. This constitutes the workflow identification step.

In the second block, identified partners negotiate their roles and responsabilities as well as the coordination of their cooperation (negotiate $(\ldots)$ ), and then interconnect to each other $(\operatorname{connect}(\ldots))$. This forms the workflow interconnection step.

After their interconnection, partners are now ready to cooperate and communicate by sending data (request $(. .)$.$) and/or receiving data (response (. .$.$) )$ 
and/or being notified (notify $(\ldots)$ ). Their communication is monitored and controlled by the contracting authority (monitor $(\ldots)$, state $(\ldots))$.

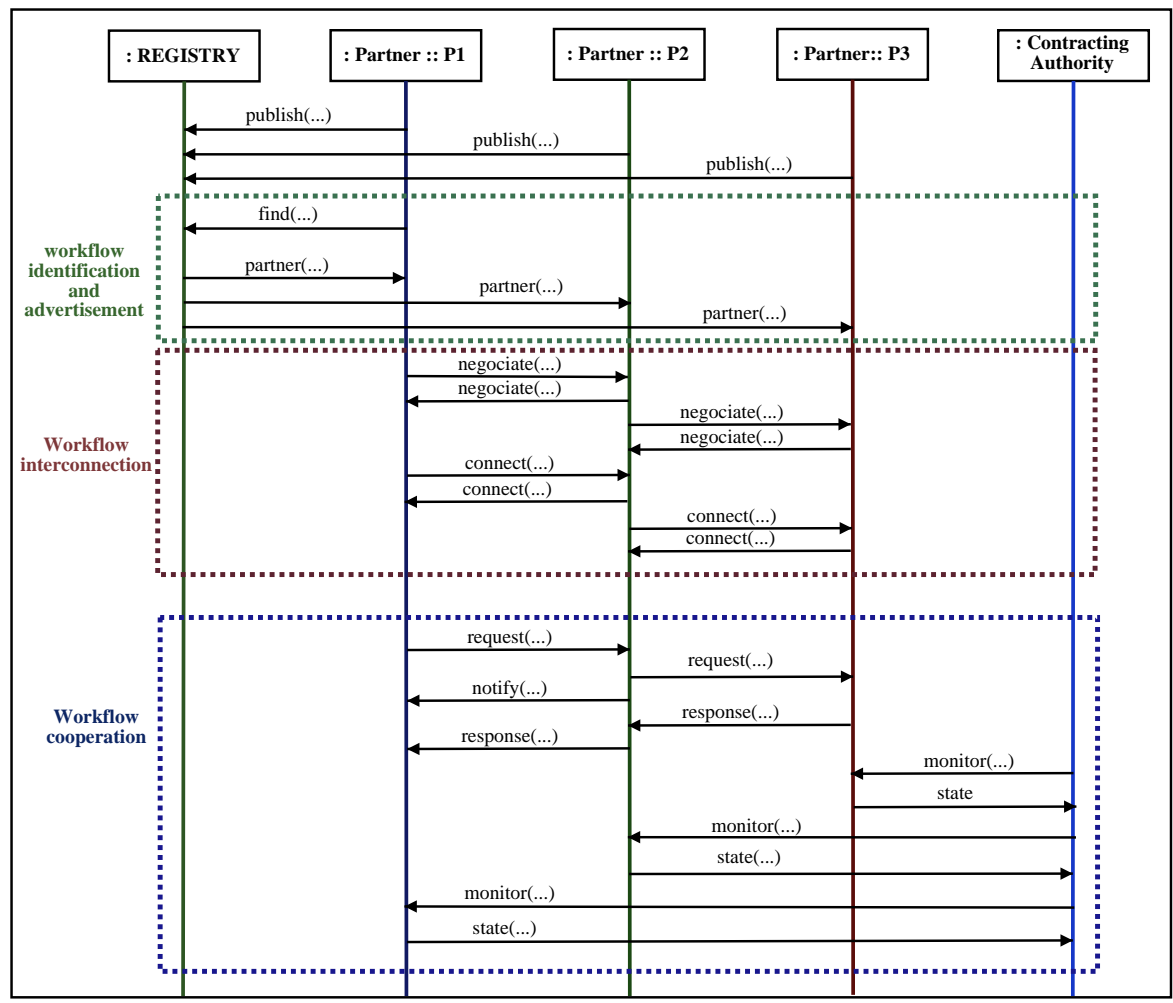

Fig. 2. Sequence Diagram of the workflow interaction

\section{Describing cooperation in inter-organizational workflows}

In this section we will discuss the question of how to describe workflow cooperation in order to meet the requirements we have presented in Section 3, including their drawbacks.

Existing approaches, like the one presented by [15], describe cooperation between workflows by specifying an inter-organizational workflow and partitioning it according to the organizations involved by private refinement of the parts based on a notion of inheritance. A drawback of this approach is the absence of privacy, since private workflows are visible or can be deduced by inheritance. 
In addition, organizations cannot modify their workflows without changing the inter-organizational workflow (i.e., the cooperation contract).

\subsection{Running example}

To illustrate the problem, consider the example, presented by Figure 3, involving four business partners (a customer, a producer and two suppliers) and illustrating the three cooperation phases mentioned above. The Customer sends an order for a product. Then it receives a notification announcing that the product has been taken into account by the producer. When the product is ready, the customer receives the delivery and then the invoice. Finally, he pays for the product he has ordered. The producer, waits for an order request. Then he searches for two suppliers to provide him with needed components in order to satisfy the received order. After that, he notifies the customer that his order is taken into account and waits for the suppliers' response. When he receives the requested components, he assembles them and delivers the product to the customer. Finally, he sends the invoice and waits for the payment. The last partners are the two suppliers which, in our example and for simplicity purposes, have exactly the same workflow. When a supplier receives an order, he begins by producing it and then checking it. If the product conforms to the specification, it will be sent to the requester, otherwise another product must be produced and the process is repeated until the order is satisfied.

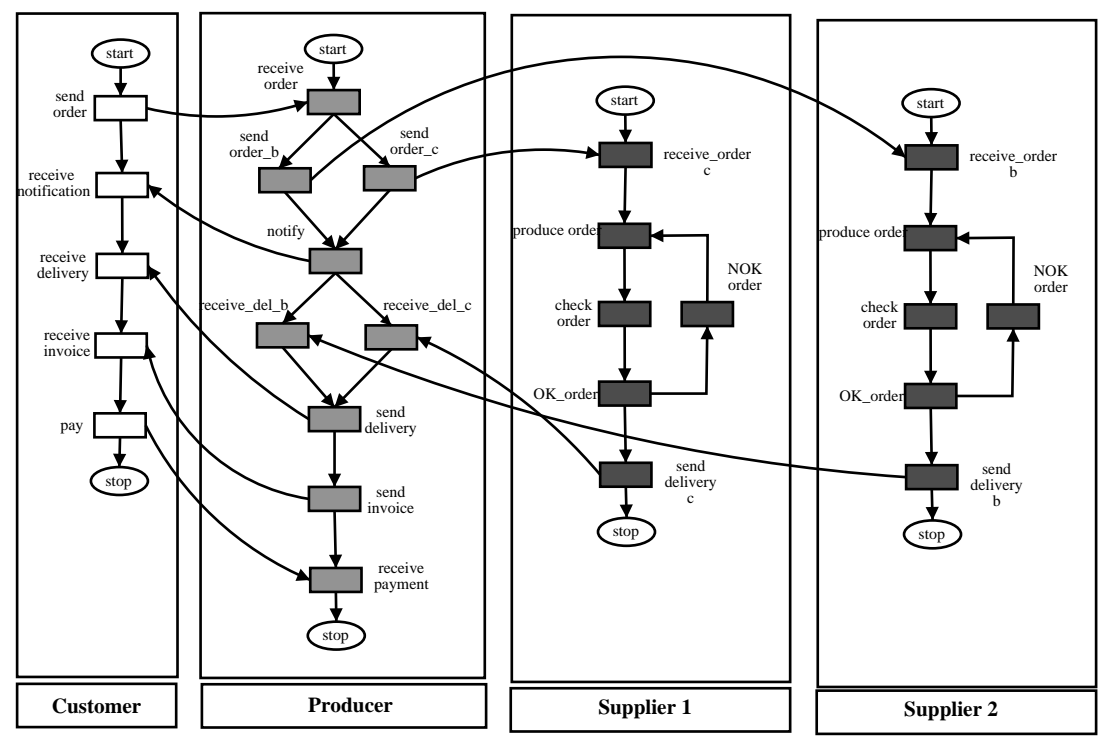

Fig. 3. An inter-organizational workflow 
As it is modeled this inter-organizational workflow does not allow any participating workflow (of a customer, the producer or the supplier) to change its internal control flows even though the role played by this participating workflow still the same. Hence, one could argue that the inter-organizational workflow is hard-wired and public. As a consequence, changing the workflow is cost-intensive.

For preserving privacy, one of our objectives is to describe the cooperation in an inter-organizational workflow without specifying the internal structure participating workflows.

\subsection{Definitions}

A cooperation within an inter-organizational workflow is considered to be the exchange between participating workflows. We use dataflows as the vehicle for providing cooperation. Two types of cooperation called Produce and Consume, are used here. A Produce is used by a workflow (A) to initiate an activity in a second workflow (B), and/or to provide input to that activity. A Consume is used by a workflow (B) to send the results of an activity (or a set of activities) to its requesting workflow (A), providing output. Although the Produce and Consume cooperation types are clearly complimentary, there is no requirement that they always be used in conjunction.

Produce and Consume cooperation types among workflows are defined by means of cooperative activities. Cooperative activities denote points in the workflow where a dataflow is produced or consumed, thereby allowing synchronization and data exchange with other workflows, as well as notification of state changes or requests of activity execution. Cooperative activities can be part of the flow structure of a workflow, just like ordinary activities, and can be of two types: producing activities or consuming activities.

Producing cooperative activity We call a producing cooperative activity each activity that produces a dataflow for an external activity that belongs to another workflow. In Figure 3, the activity send ${ }_{o} r d e r$ is a producing cooperative activity.

Consuming cooperative activity We call a consuming cooperative activity, each activity that consumes a dataflow from an external activity that belongs to another workflow. In figure 3 , the activity receive ${ }_{n}$ otification is a consumer cooperative activity.

Cooperative activity We call a cooperative activity a cooperative consuming and/or producing activity.

\subsection{Internal, cooperative and public processes}

The important things in the example of Figure 3 are the cooperative activities and the interactions (control flow) between them rather than the non-cooperative 
activities or the control flow between them. Many items depicted in Figure 3 are not important (necessary) vis-à-vis to the description of cooperation. We quote among others the following information in workflow of the supplier 1 :

- the produce_order, check_order, OK_order and NOK_order activities,

- the control flows between these activities.

If we hide this type of information, we can provide more flexibility to the supplier 1 to perform the activities produce_order, check_order, OK_order and NOK_order separately or within one or two activities without changing the interactions with the producer. Moreover, this preserves privacy since it hides the internal structure of the workflow.

In order to be able to hide internal structures of participating workflows, we propose to use the notion of public process representing one view, among others, of a participating workflow. As an example, Figure 4 depicts public processes of the workflows supplier 1 and producer. The public process of the producer is composed of two virtual activities connected to two cooperatives activities (send_order_c and receive_del_c). The public view of supplier_1 is also composed of two virtual activities connected to two cooperative activities (receive_order_c and send_delivery_c). The connection between the virtual activities describe the cooperation between these two workflows (i.e., the cooperation policy).

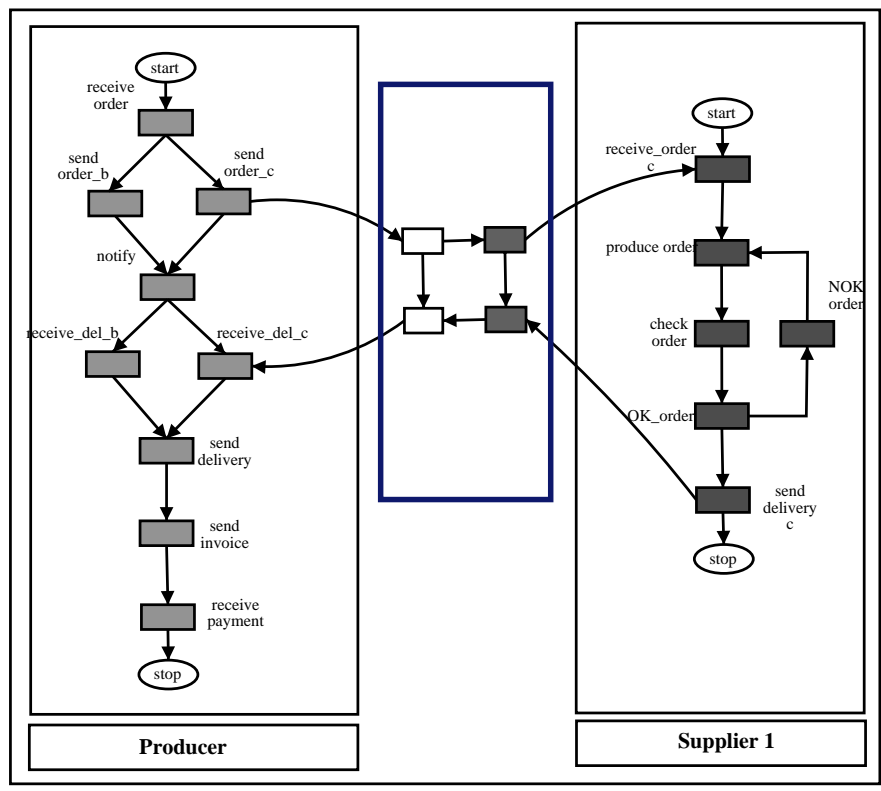

Fig. 4. Public vs. internal processes 
By representing a public process, we hide their internal structures and we focus on cooperation between the workflows. In fact, by Figure 4, only virtual activities and the control flows between them are visible. Connections between virtual and cooperative activities are not. Given a virtual activity, partners can not identify which cooperative activity will be performed. It is up to the workflow owner to decide which cooperative activity will be eventually performed.

As a result, inter-organizational workflow cooperation presented in Figure 4, composed of virtual activities and their connection, represents a cooperation contract between the participating workflows. This contract allows several cooperation scenarios, in which internal workflow structures are changed, and privacy is preserved. It allows participating organizations to adapt and modify their workflows without changing their cooperation.

For that reason the producer can change its internal structure (see left part of Figure 5) without changing its role in the cooperation (the public process is the same). In the same way, the supplier can change its internal structure (see right part of Figure 5).
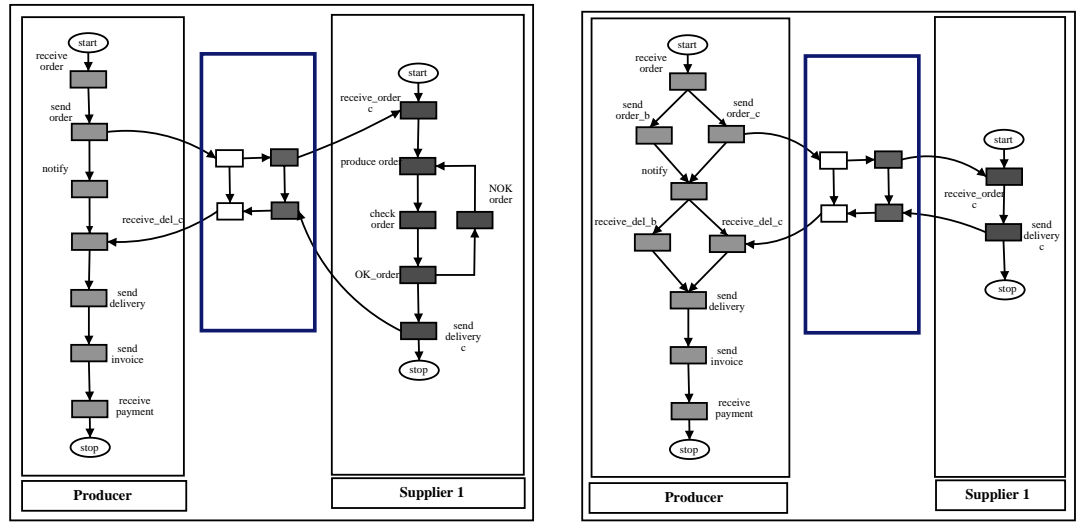

Fig. 5. Producer and Supplier change their internal processes

For the three different scenarios presented in Figures 4 and 5, participating workflows have the same public processes and the same interconnection between them, representing the cooperation contract.

Figure 6 illustrates the cooperation architecture involving partners cooperating via their public processes, where the communication is ensured by a middleware.

In Figure 6, several organizations with complementary competencies want to cooperate and carry out a work that is not within the range of only one of them without revealing their proper skills. To do so, these organizations hide their internal processes and generate one or more public processes to communicate 
with the other partners with an inter-visibility as tiny as cooperation needs. Finally, the communication will be ensured by a middleware.

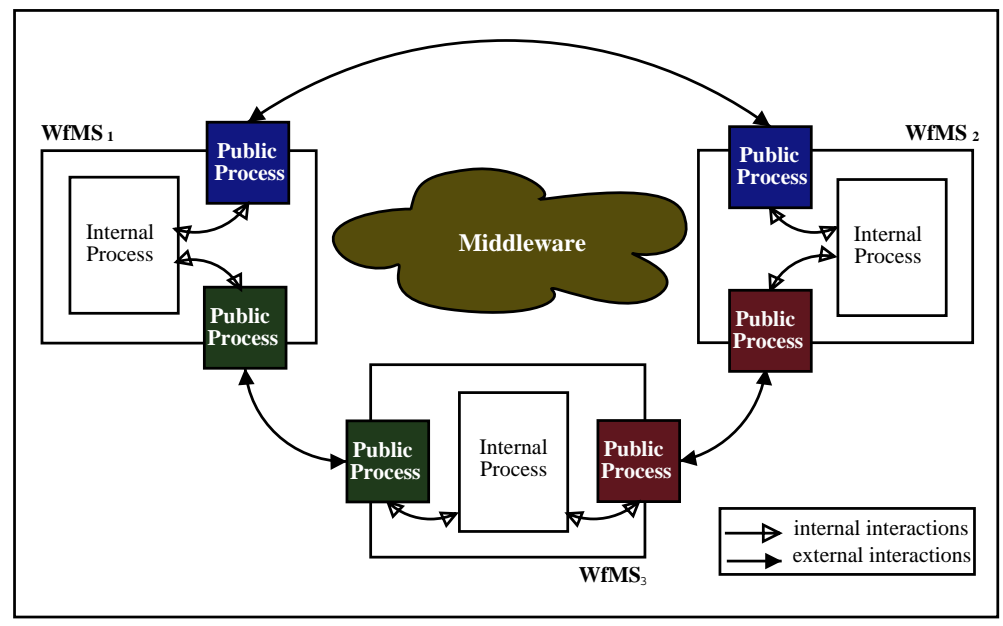

Fig. 6. Cooperation Architecture

We have introduced by this example the notion of public processes. In the following section we present its design process.

\subsection{Definition process of public processes}

In this section, we present how the cooperative process can be deduced from the internal one as well as how a public process can be defined in order to depict a selected view of the cooperative one.

Cooperative processes First, each partner defines its internal process including its know-how where all dataflows and internal (cooperative and non cooperative) activities as well as their control flows are specified (or identified). An internal process can abstract or specialize a pre-established process.

In our example, at the beginning, none of the business partners knows the other partners it will collaborate with. Thus, each of them models its proper internal process specifying all steps needed to accomplish its services independently of the others. The customer, producer, first and second supplier's internal processes are illustrated in figure 7.

Then, for each internal process, we can identify a sub-process where all activities that will produce and/or consume dataflows (cooperative activities) as well as their coordination are extracted and all extra and unnecessary information are hidden. We call such a sub-process a cooperative process. The cooperative 

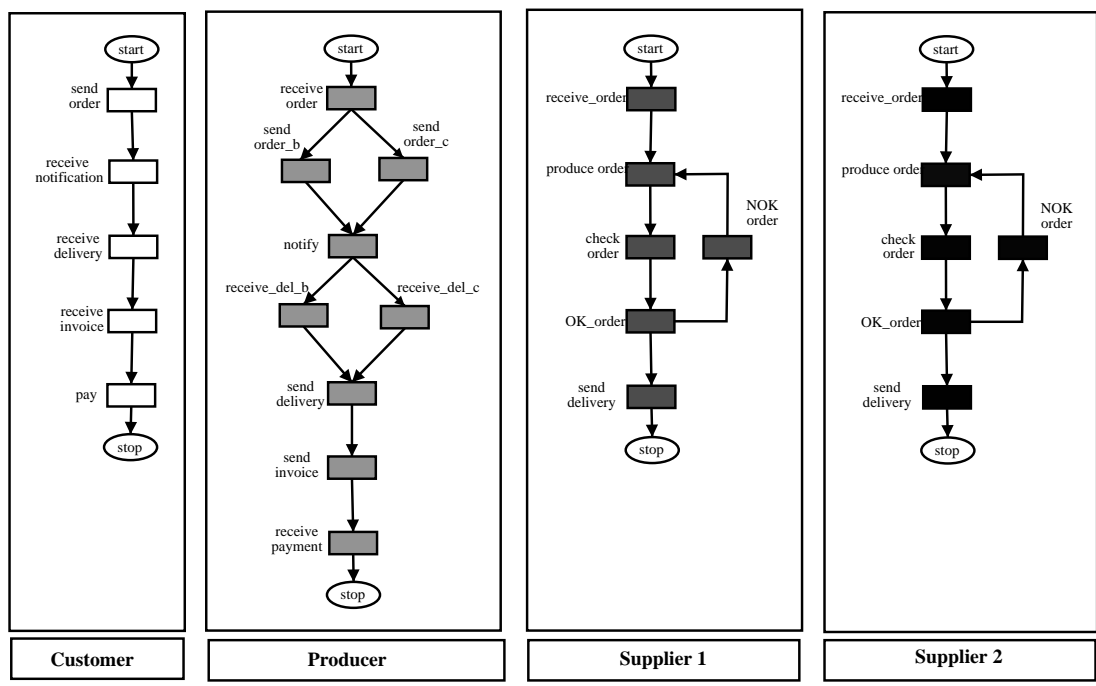

Fig. 7. Customer, Producer and Suppliers's Internal Processes

process is the "minimal" connected and compacted sup-process that contains all cooperative activities ${ }^{3}$.

To pass from an internal workflow to a cooperative one, we begin with finding the minimal connected workflow and then transform it into a cooperative workflow using a compacting procedure. First, we determine a minimal connected workflow composed of all the cooperative activities as well as the internal activities between them ensuring the connectivity of the whole workflow. Second, we use a compacting procedure to transform the the "minimal" connected process into a cooperative one in which non cooperative activities act just as connection activities.

In our example, the producer can cooperate with three partners: a partner that requests for an order (customer) and two others to construct the received order components (two suppliers). At this step, he extracts the different cooperative activities to form the corresponding cooperative process allowing the cooperation with the other partners. The resulting process is composed of the follwing activities: the activities receive_order, notify, send_delivery, send_invoice, receive_payment to allow cooperation with the customer, and the activities send_order $r_{b / c}$, receive_del $_{b / c}$ to permit cooperation with the two suppliers.

The customer needs only one partner to cooperate with : a partner for the production of a specified product. He begins by building his internal process and then extracts all cooperative activities to form the cooperative process which,

\footnotetext{
${ }^{3}$ See appendix for formal definition of a cooperative process
} 
in our case, is composed of five activities : send_order, receive_notification, receive_delivry, receive_invoice and the pay activities and the.

Finally, the same rules are applied to the suppliers's workflows. In our example, the suppliers have only two cooperative activities allowing them to receive and then deliver an order : receive_order and send_delivery in order to cooperate with the producer.

Figure 8 presents the cooperative workflows corresponding to the four partners.

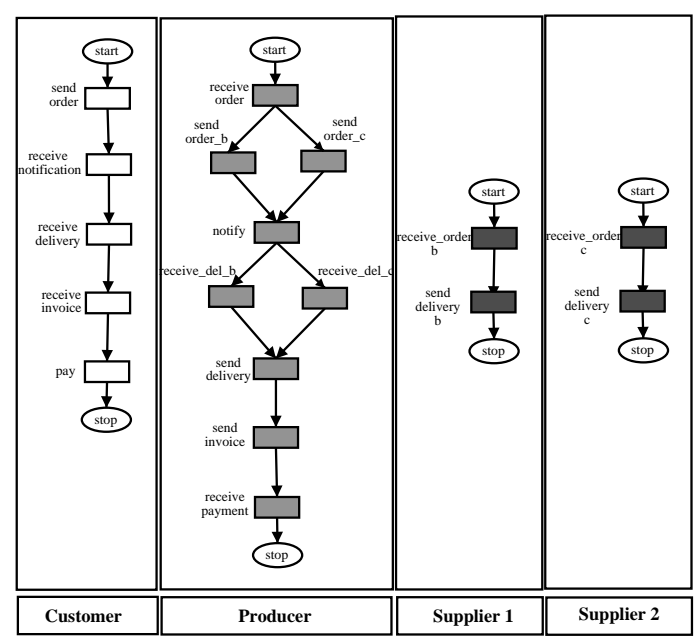

Fig. 8. Partners' Cooperative Workflows

Public processes After determining the internal and cooperative processes, public processes are created for one of the following purposes: A workflow wants to expose some activities or it wants to access activities of workflows of other organizations.

A public process is a public view of the cooperative process. A cooperative process can expose many public processes at the same time. Each one is used to define the cooperative with one partner. A public process consists of virtual activities which represent a subset of the cooperative process activities. Virtual activities are not supposed to produce or consume output/input. Nevertheless, they are supposed to transfer output/input data to/from other workflows. Similarly they are not meant to be executed by local role.

In the following we present how public processes can be deduced from the cooperative ones using the notion of cooperation policy. The cooperation policy establishment process we propose in this paper integrates the participants' roles during cooperation, the dataflow that will be passed from one partner to 
another and the participant's public processes that will be exposed and accessed by external organizations. Given a set of organizations with complementary competencies, we describe their interactions in terms of a cooperation policy, which is a set of rules between participants defined in terms of dataflow and access contracts, and workflows public process definition.

In order to establish a cooperation policy, we propose a three phases process: a dataflow contract establishment to express dataflows to be exchanged between partners, an access contract establishment to express allowed activities one partner can execute on its behalf by an external partner and workflow public process definitions.

After determining the different activities participants' workflow can be executed on its behalf within an external workflow, each partner specifies the different dataflows to exchange with the other partners. The result of this step is a set of rules associating, in a peer to peer manner, the partners with the data they can send to each other. We call this set of rules a dataflow contract. In our example, the dataflow contracts are illustrated by figure 9

\begin{tabular}{|c|c|c|c|}
\hline \multicolumn{2}{|c|}{ Customer-Producer Dataflow Contract } & \multicolumn{2}{|c|}{ Producer-Supplier $_{1 / 2}$ Dataflow Contract } \\
\hline Customer & Producer & Producer & Supplier/2 \\
\hline $\begin{array}{l}\text { * order_a } \\
* \text { payment }\end{array}$ & $\begin{array}{l}* \text { notification } \\
* \text { delivery_a } \\
* \text { invoice }\end{array}$ & $*$ Order $_{\mathrm{b} / \mathrm{c}}$ & * Delivery $_{\mathrm{b} / \mathrm{c}}$ \\
\hline
\end{tabular}

Fig. 9. Partners Dataflow Contracts

The objective of the access contract establishment is to determine, for each partner, the set of external activities (activities executed by external workflows on its behalf) it can execute as well their coordination. This consists of identifying the roles of the different identified partners, as well as the coordination of their cooperative activities. Hence, the result is a set of rules describing the responsibilities and the roles played by each partner in the cooperation. We call this set of rules an access contract. In Figure 10, we give the access contracts between the producer and the customer, and between the producer and the two suppliers.

Based on the access contracts, each partner defines the visibility levels of its workflow to the other partners. Hence, many views permitting to cooperate without revealing its know-how are generated. This allows providing each partner with only information it needs to know by concealing all details and extrainformation. This reduces the inter-visibility to be as little as required for the cooperation.

In our example, the customer cooperates only with the producer, hence it generates only one public process composed of five virtual activities. The producer generates three public processes, the first is composed of five virtual activities to interconnect to the customer and the two other ones are identical and composed of two virtual activities in order to permit the sent of an order and the recep- 


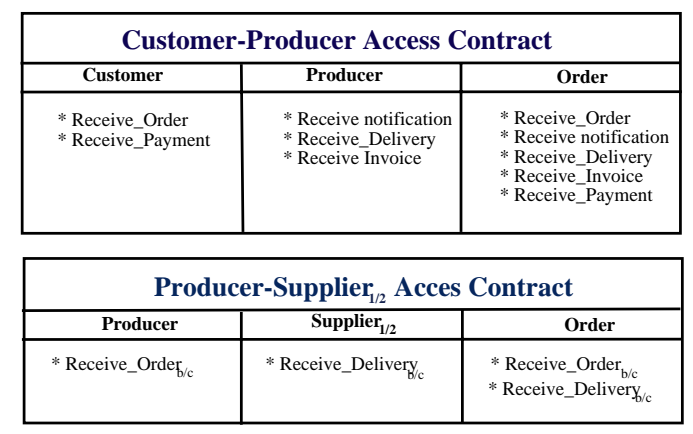

Fig. 10. Partners Access Contracts

tion of the correspondent delivery. Figure 11 shows the partners' public views workflows.

\begin{tabular}{|c|c|c|c|c|}
\hline customer & \multicolumn{3}{|c|}{ producer } & supplier1,2 \\
\hline Producer View & Customer View & Supplier1 View & Supplier2 View & Producer View \\
\hline ק & T & & & \\
\hline 古 & t & 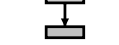 & t & \\
\hline 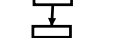 & ד & & & \\
\hline$\frac{1}{t}$ & 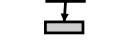 & & & \\
\hline
\end{tabular}

Fig. 11. Partners' Public Views

After defining internal, cooperative, and public processes, the four partners are ready to interconnect to each other and then cooperate via their public processes (see Figure 12). The communication between them will be ensured by a middleware.

The interconnection of the different partners is illustrated in Figure 13, where every partner is composed of three main components : the public processes for the cooperation with other partners, the internal process containing the know-how of the organization and the partner WfMS that will execute the corresponding workflow.

\section{$6 \quad$ Implementation Issues}

Discussing details of implementation is not in the intended scope of this paper. Currently we are implementing a prototype of our workflow cooperation platform (see Figure 14). Existing workflow management systems (WfMS) can plug into our platform. This operation can be done if the WfMS fulfills two conditions. 


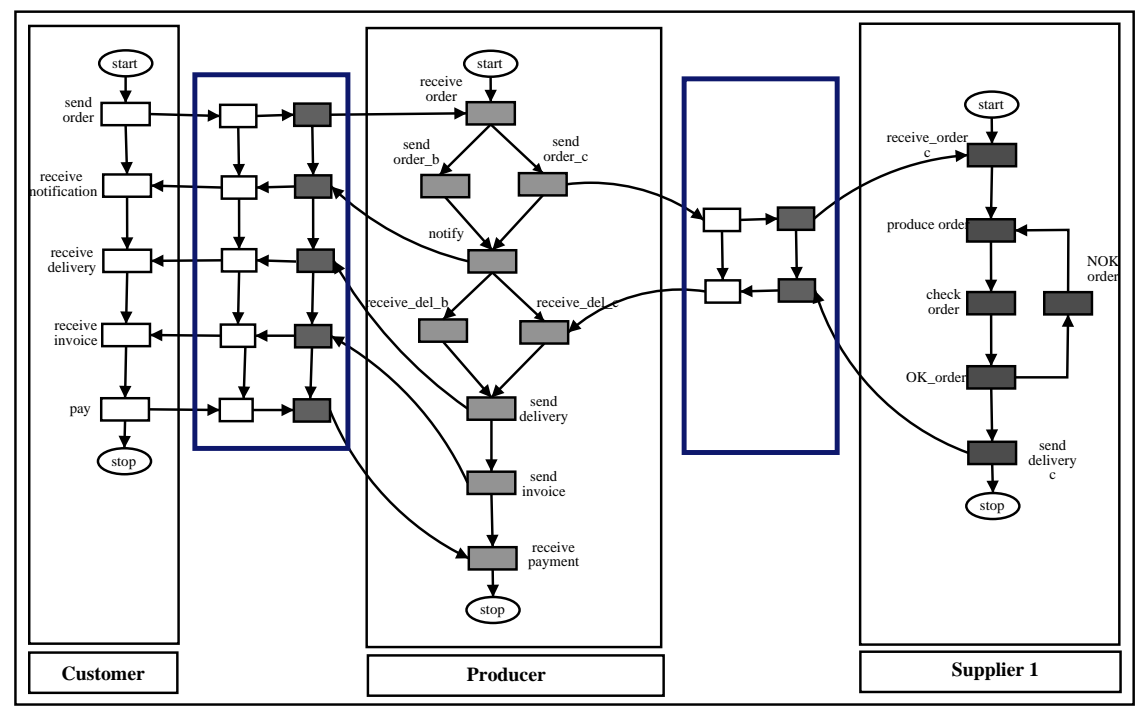

Fig. 12. Partners' Interconnection

First, the WfMS can call external applications (e. g. programs, Web services, etc.). Second, the WfMS allow external applications to invoke any step within a workflow it manages. To adapt incoming and outgoing invocations, we use wrappers.

Our implementation relies on the development of patterns for cooperation. Actually, a workflow may be related to proxy and adapter patterns. The wrappers shown in Figure 14 represent one proxy and one adapter for each workflow. While the proxy pattern limits accesses to workflow resources (data access rights, method visibility, visibility of business process events), the adapter pattern provides a new interface to the adapted workflow (ability to exchange data, coordination ability, ability to control interaction consistency).

The role of wrappers is interfacing of a workflow with the registry and the contracting authority. Namely, the wrapper serves a workflow by adapting its actions (which depend on the characteristics of the system it holds). The actions performed can be data management operations and coordination actions. Interactions between workflows are controlled by the contracting authority. Only interactions that satisfy negotiated cooperation policies are accepted.

One of the goals of our work is to allow workflows to cooperate through partial visibility of their resources (e.g. data sharing, group awareness). In addition, it is necessary to provide to cooperating workflows means to coordinate their actions and to work in an autonomous way while being aware of what occurs in the virtual organization. For this reason, each workflow has to provide events allowing other services to know the state of its execution and its private data. 


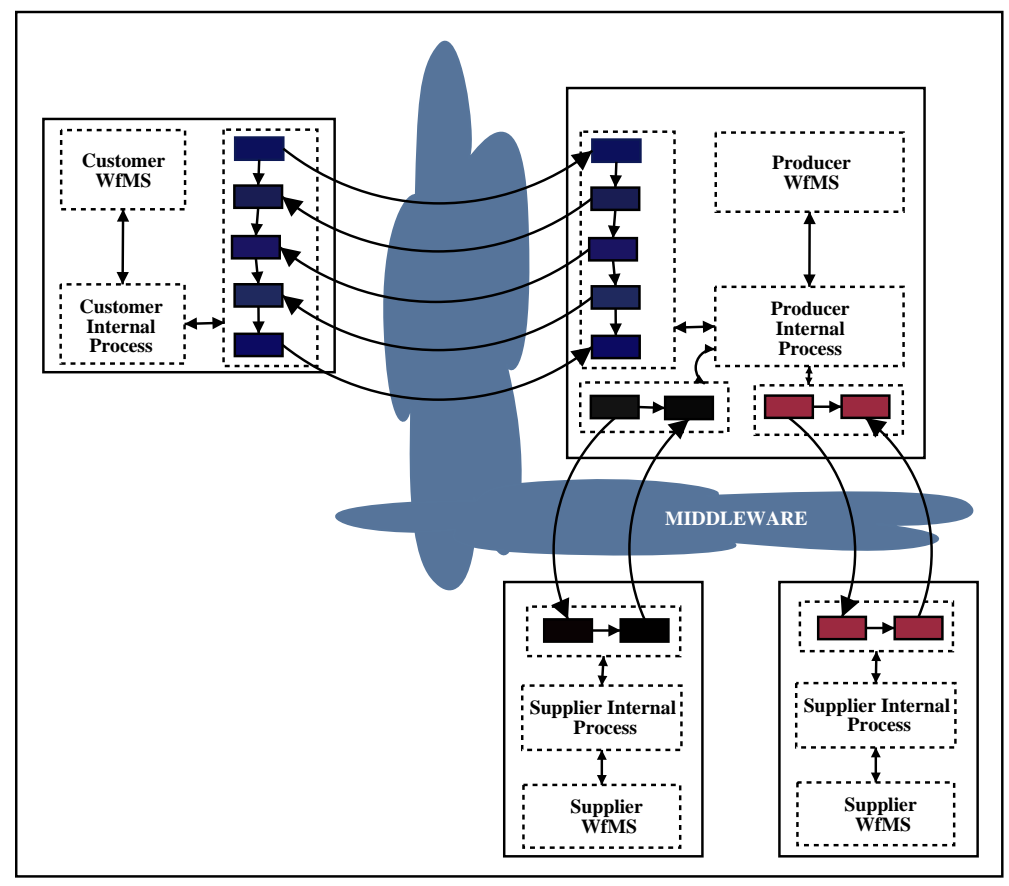

Fig. 13. Customer, Producer and Suppliers interaction scenario

Since each workflow is interested in specific information, it is beneficial to control the visibility of workflow events. This control is provided by the adapter.

So far, we have developed two wrappers for two existing workflow management systems : XFlow ${ }^{4}$ and OSWorkflow ${ }^{5}$. In the following, we present both of the WfMS's and then describe the cooperation scenario between them. XFlow is a pure J2EE platform for building, executing and managing business processes and workflows. It runs within an EJB and servlet container. JBoss 4.0 (with bundled Tomcat) is the container used in our implementation. The architecture of XFlow supports distributed and parallel processes within an organizations' firewall as well as across organizational boundaries.

The second workflow management system we have used is called OSWorkflow and is a Java based open source project.

We mention here that both of the WfMS's used fulfill the two conditions of cooperation which are the capability of calling external applications and allowing these ones to invoke any step within a workflow they manage.

The communication between the two systems is supported by a Web service middleware. And in order to preserve the systems privacy and limit accesses to

\footnotetext{
${ }^{4}$ XFlow: http://xflow.sourceforge.net/

${ }^{5}$ OSWorkflow: http://www.opensymphony.com/osworkflow/
} 


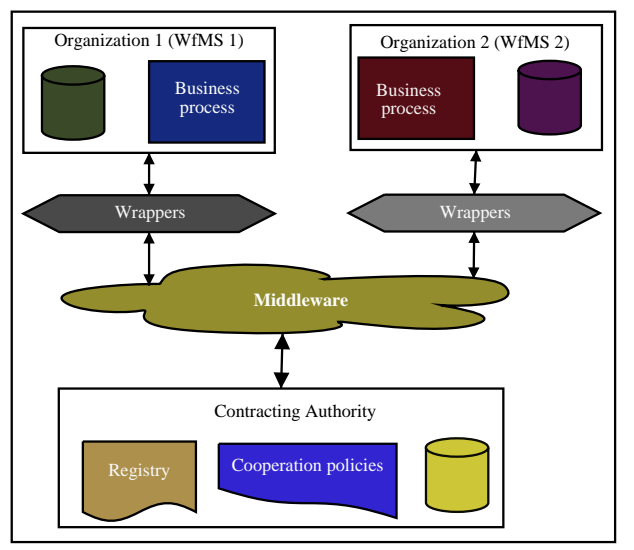

Fig. 14. A platform for inter-organizational workflow

workflow resources (data access rights, method visibility, etc.), we have developed two wrappers that play the proxy role for both of them. These wrappers are in the form of two simple web services, deployed in WS containers (Tomcat), that launch some activities of the workflows. Figure 15 shows an overview of the WfMS's deployment.
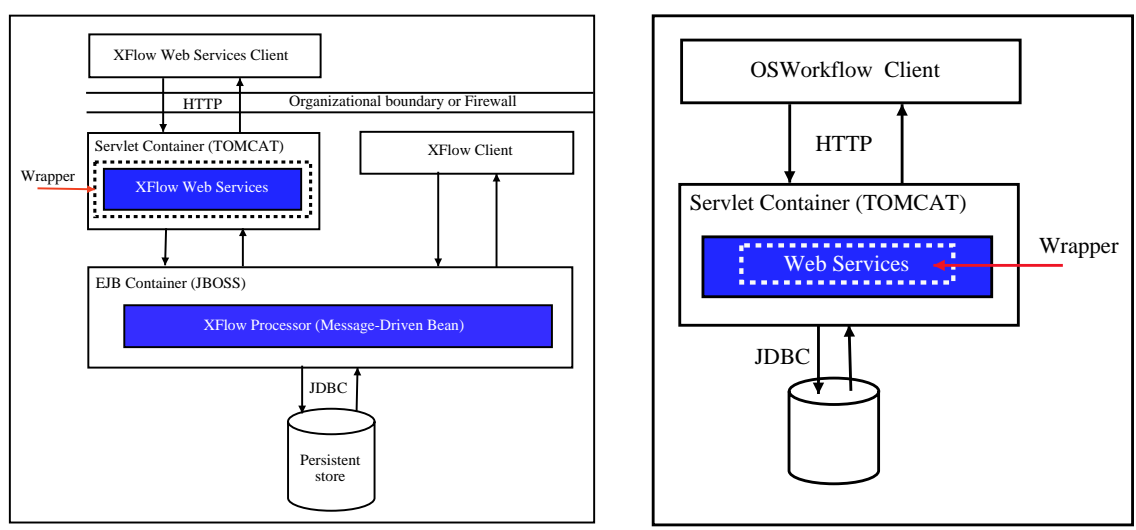

Fig. 15. XFLOW and OSWorkflow deployment

Figure 16 shows the communication scenario between the two WfMS's we have inter-connected. The figure is composed of three major blocks. The first contains the internal workflow of the producer, the second contains the internal 
workflow of the customer, and finally, we find the public views used by the partners to cooperate. In order to run the example, the first step consists in launching the workflow management systems on the both sides. Then, the producer and the customer cooperate and each one calls the activities it is authorized to invoke via Web services.

After cooperating, both of the workflow systems progress independently.

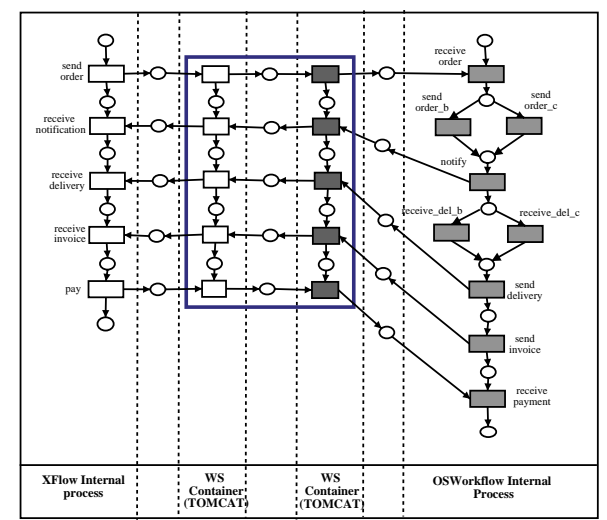

Fig. 16. Communication scenario

\section{Conclusion and Future Work}

In this paper we presented important steps to provide support for inter-organizational workflows. The relevance of inter-organizational workflows is best seen when considering emerging virtual organizational forms, consisting of geographically dispersed teams and their respective business processes. Our approach allows for partial visibility of workflows and their resources, thus providing powerful ways for inter-organizational workflow configuration. Varying degrees of visibility of workflows enable organizations to retain required levels of privacy and security of internal workflows. Furthermore, the view concept provides a high degree of flexibility for participating organizations, since internal structures of collaborative workflows may be adapted without changes in the inter-organizational workflows. Currently we are in the process of finalizing building a prototype system where our ideas are implemented, validated, and tested.

This paper presents an approach to inter-organizational workflows. Many open issues have to be addressed in our future work. For example, the question here is what are the necessary properties local workflows have to satisfy in order to be consistent with the cooperation? Examples of these properties are sound- 
ness and correctness criteria. What are the mechanisms utilized for semantic registries? More research is requited on those issue.

\section{References}

1. Davidow, W., Malone, M.: The virtual corporation. Harper BusinessHarper Business (1992)

2. Gornev, V., Rarassov, V., Soenen, R., Tahon, K.: Virtual enterprise : Reasons, sources and tools. In: MCPL'97, Campinas, Brazil (1997) 53-58

3. Byrne, J.A.: The virtual corporation. Business Week 31 (1993) 36-39

4. van der Aalst, W.M.P.: Loosely coupled interorganizational workflows: Modeling and analyzing workflows crossing organizational boundaries. Information and Management 37 (2000) 67-75

5. Allen, R.: Workflow: An introduction. In Fisher, L., ed.: Workflow Handbook. Future Strategies, Lighthouse Point, FL (2001) 15-38

6. Grefen, P., Aberer, K., Hoffner, Y., Ludwig, H.: Crossflow: Cross-organizational workflow management in dynamic virtual enterprises. International Journal of Computer Systems Science \& Engineering 15 (2000) 277-290

7. Dijk, A.V.: Contracting workflows and protocol patterns. In: Proceedings BPM, Eindhoven, The Netherlands (2003)

8. Alonso, G., Fiedler, U., Hagen, C., Lazcano, A., Schuldt, H., Weiler, N.: Wise: Business to business e-commerce. Proceedings of 9th International Workshop on Research Issues on Data Engineering: Information Technology for virtual Enterprises (1999)

9. Lazcano, A., Alonso, G., Schuldt, H., Schuler, C.: The wise approach to electronic commerce. International Journal of Computer Systems Science \& Engineering, special issue on Flexible Workflow Technology Driving the Networked Economy vol. 15 (2000)

10. Perrin, O., Godart, C.: A model to support collaborative work in virtual enterprises. Data \& Knowledge Engineering 50 (2004) 63-86

11. Andrews, T., Curbera, F., Dholakia, H., Goland, Y., Klein, J., Leymann, F., Liu, K., Roller, D., Smith, D., Thatte, S., Trickovic, I., Weerawarana, S.: Business process execution language for web services, version 1.1 (2003)

12. van der Aalst, W.M.P.: Process-oriented architectures for electronic commerce and interorganizational workflow. Inf. Syst. 24 (1999) 639-671

13. Muth, P., Wodtke, D., Weissenfels, J., Angelika Kotz Dittrich, G.W.: From centralized workflow specification to distributed workflow execution. Journal of Intelligent Information Systems (1998) 10(2):159-184

14. van der Aalst, W.M.P.: Interorganizational workflows: An approach based on message sequence charts and petri nets. Systems Analys is - Modelling - Simulation 34 (1999) 335-367

15. van der Aalst, W.M.P., Weske, M.: The p2p approach to interorganizational workflows. In: Proceedings of the 13th International Conference on Advanced Information Systems Engineering, Springer-Verlag (2001) 140-156

16. Zhao, J.L.: Workflow management in the age of e-business. In: Tutorial at the 35th Hawaii International Conference on System Sciences, Waikoloa, Hawaii (2002)

17. Grefen, P., Aberer, K., Hoffer, Y., Ludwig, H.: Crossflow: Cross-organizational workflow management for service outsourcing in dynamic virtual enterprises. IEEE Data Engineering Bulletin 24 (2001) 52-57 
18. Christensen, E., Curbera, F., Meredith, G., Weerawarana, S.: Web services description language (wsdl) version 1.1. Technical report, W3C Note (2001)

19. Leymann, F.: Web services flow language (wsfl 1.0). Technical report, IBM (2001 (cf. http://www-4.ibm.com/software/solutions/webservices/pdf/WSFL.pdf))

20. Thatte, S.: Xlang - web services for business process design. (2001)

21. Arkin, A.: Business process modeling language(bpml). Working draft 0.4, BPMI (2001 (cf. http://www.bpmi.org))

22. A. Banerji et al: Web services conversation language (wscl) 1.0. (2002)

23. ebXML: http://ebxml.org. (2002)

24. Workflow Management Coalition: Interface 1 : Process definition interchangeprocess model. doc. no. wfmc tc 1016-p, version 1.1 (official release). Technical report (1999)

25. Gerhard, K., Retschitzegger, W., Bernauer, M., Kappel, G.: Specification of interorganizational workflows - a comparison of approaches. Technical Report 08/02, Institute of Software Technology and Interactive Systems, Business Informatics Group, Vienna University of Technology (2002)

26. Russell, N., ter Hofstede, A., Edmond, D., van der Aalst, W.: Workflow data patterns. Technical Report FIT-TR-2004-01, Queensland University of Technology, Brisbane (2004)

27. Mers, M., Liberman, B., Muller-Jones, K., Lamersdorf, W.: Interorganisational workflow management with mobile agents in cosm. In: Proceedings of PAAM96 Conference on the Practical Application of Agents and Multiagent Systems. (1996)

28. Tata, S.: Policies for cooperative virtual teams. In: International Conference on Coordination Languages and Models, COORDINATION'02, York, UK (2002)

29. Baina, K., Tata, S., Benali, K.: A model for process service interaction. In van der Aalst, W.M.P., ter Hofstede, A., Weske, M., eds.: Business Process Management: International Conference. LNCS 2678, Eindhoven, The Netherlands, SpringerVerlag Heidelberg (2003) 261-275

30. Tata, S., Boughzala, I.: Modeling contribution for virtual enterprise support. In: 12th IEEE International Workshops on Enabling Technologies, Linz, Austria, IEEE Computer Society (2003) 165-170

31. Kauffman, R., Walden, E.: Economics and electronic commerce: Survey and research directions (2001)

32. Ba, S., Stinchcombe, M., Whinston, A.B., Zhang, H.: Trusted third parties in the electronic marketplace. In Haseman, D., Nazareth, D., eds.: Proceedings of the Fifth Americas Conference on Information Systems, Milwaukee, WI (1999) 


\section{Appendix}

In this appendix we present how to pass from an internal workflow to a cooperative one in two steps. We begin with finding the minimal connected workflow and then transform it into a cooperative workflow using a compacting procedure.

In the first step, we start from an internal workflow. Based on an incremental construction, we determine a minimal connected workflow composed of all the cooperative activities as well as internal activities between them ensuring the connectivity of the whole workflow.

The next step consists of hiding all extra activities that will not play a connection role in the workflow. This step will keep only internal activities that will act just as connectors between cooperatives activities that are called connection activities.

To illustrate how to pass from an internal workflow to a cooperative one, consider the example presented by Figure 17, showing three workflows : an internal workflow, the corresponding minimal connected wokflow and finally the compacted workflow. The first workflow presents the know-how of the organization and contains all required activities as well as their coordination. We distinguish two kinds of activities : internal ones presented by white rectangles $\left(t_{1}, t_{2}, t_{4}\right.$, $t_{5}, t_{9}, t_{12}, t_{13}$ and $\left.t_{14}\right)$, and cooperative ones presented by black restangles $\left(t_{3}\right.$, $t_{6}, t_{7}, t_{8}, t_{10}$ and $t_{11}$ ) (figure $17(\mathrm{~b})$ ). The second workflow presents the minimal connected workflow where we only retain the cooperative acitivities and internal ones that ensure the connectivity of the workflow $\left(t_{1}, t_{2}, t_{4}, t_{5}\right.$ and $\left.t_{9}\right)$. This workflow is then compacted in order to eliminate all extra information and activities.

\subsection{Minimal connected workflow}

Definition 1. A Workflow $W(P, T, F)$ is determined by :

$$
\begin{aligned}
& \text { - a finite set } P=\left\{p_{1}, p_{2}, \ldots, p_{n}\right\} \text { of places } \\
& \text { - a finite set } T=\left\{t_{1}, t_{2}, \ldots, t_{m}\right\} \text { of transitions }(P \cap T=\emptyset) \\
& \text { - a set of arcs } F \subseteq(P \times T) \cup(T \times P)
\end{aligned}
$$

The set of input (output) places for a transition $t$ is denoted $\bullet$ t ( $\bullet$ ). The set of transitions sharing a place $p$ as output (input) place is denoted $\bullet p(p \bullet)$.

The set $T=T_{\text {coop }} \cup T_{\text {int }}$ where $T_{\text {coop }}$ is the set of cooperative activities and $T_{\text {int }}$ is the set of internal activities.

Definition 2. A petri net $W=(P, T, F)$ is a WF-net (Workflow net) if and only if [14]:

1. Whas two special places: $i$ and o. Place $i$ is a source place $: \bullet i=\emptyset$. Place $o$ is a sink place : $o \bullet=\emptyset$.

2. If we add a transition $t^{*}$ to $W$ which connects place $o$ with $i$, and a transition $t_{j}$ which connects $i$ with $p_{j}$ for each $p_{j} \in \ldots$, then the resulting Petri net is strongly connected. 


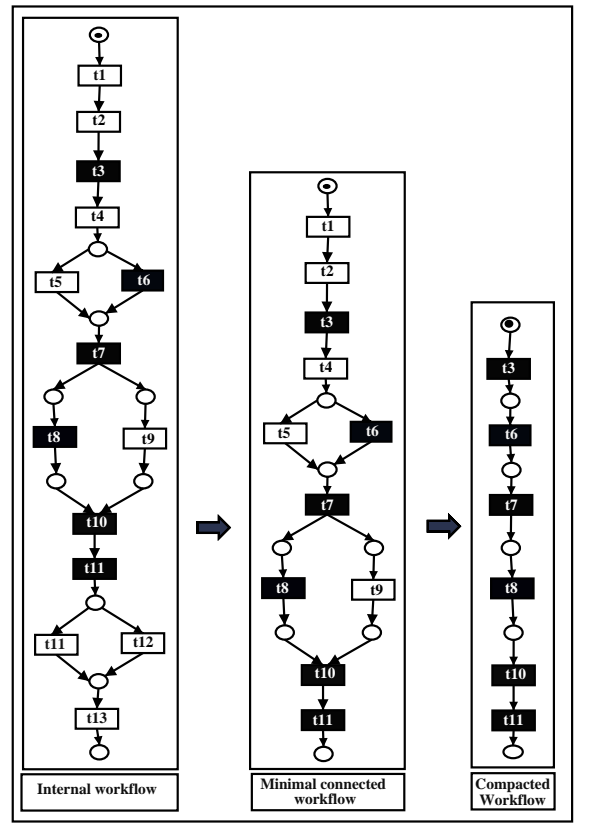

Fig. 17. Internal-Minimal-Compacted workflows

Definition 3. Let $W(P, T, F)$ be a WF-net, $T=T_{\text {coop }} \cup T_{\text {int }}$. A minimal cooperative connected workflow $W_{c}\left(P_{c}, T_{c}, F_{c}\right)$ is determined by:

$-T_{c} \subseteq T$

$-T_{\text {coop }} \subseteq T_{c}$

$-P_{c} \subseteq P$

- $W_{c}$ is minimal connected (i.e, $\nexists W_{c}^{\prime}\left(P_{c}^{\prime}, T_{c}^{\prime}, F_{c}^{\prime}\right) \neq W_{c}$ where $W_{c}^{\prime}$ is connected, $T_{\text {coop }} \subseteq T_{c}^{\prime}, T_{c}^{\prime} \subseteq T_{c}, F_{c}^{\prime} \subseteq F_{c}$ and $\left.P_{c}^{\prime} \subseteq P_{c}\right)$

\subsection{Cooperative workflow}

After determining the minimal connected workflow, the next step consists of simplifying the workflow by removing all unnecessary information and hiding activities and flows that don't play any role in the cooperation or the maintaining of the workflow connectivity. 
Definition 4. Let $t_{i}, t_{j} \in T$ and $p_{i j} \in$ $P . t_{i}$ and $t_{j}$ are sequent and denoted $\operatorname{seq}\left(t_{i}, t_{j}, p_{i j}\right)$ if and only if

$-\bullet p_{i j}=t_{i}$
$-p_{i j} \bullet=t_{j}$
$-t_{i} \bullet=p_{i j}$
$-\bullet t_{j}=p_{i j}$

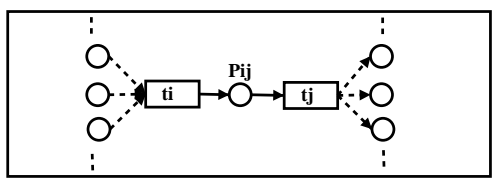

Definition 5. Let $t_{i}, t_{j} \in T$ and $p_{i j}^{i}, p_{i j}^{o}$ $\in P . t_{i}, t_{j}$ are alternative and denoted $\operatorname{alt}\left(t_{i}, t_{j}, p_{i j}^{i}, p_{i j}^{o}\right)$ if and only if

$-t_{i}, t_{j} \in p_{i j}^{i} \bullet$

$-t_{i}, t_{j} \in \bullet p_{i j}^{o}$

$-\bullet t_{i}=\bullet t_{j}=p_{i j}^{i}$

$-t_{i} \bullet=t_{j} \bullet=p_{i j}^{o}$

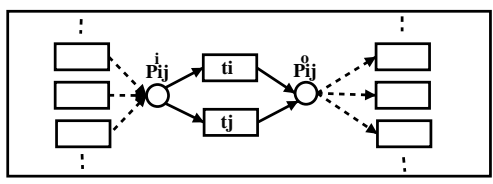

Definition 6. Let $t_{i}, t_{j}, t_{k}, t_{l} \in T$ and $p_{k i}$, $p_{i l}, p_{k j}, p_{j l} \in P . t_{i}, t_{j}$ are synchronized and denoted $\operatorname{sync}\left(t_{i}, t_{j}, t_{k}, t_{l}, p_{k i}, p_{i l}, p_{k j}, p_{j l}\right)$ if and only if

$$
\begin{aligned}
& -\bullet p_{k i}=t_{k}, p_{k i} \bullet=t_{i} \\
& -\bullet p_{k j}=t_{k}, p_{k j} \bullet=t_{j} \\
& -\bullet p_{i l}=t_{i}, p_{i l} \bullet=t_{l} \\
& -\bullet p_{j l}=t_{j}, p_{j l} \bullet=t_{l} \\
& -\bullet t_{i}=p_{k i}, t_{i} \bullet=p_{i l} \\
& -\bullet t_{j}=p_{k j}, t_{j} \bullet=p_{j l}
\end{aligned}
$$

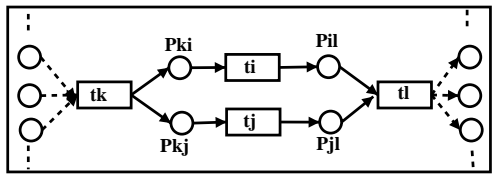

\subsection{Definition procedure of cooperative workflow}

Given a minimal cooperative connected workflow $W_{c}\left(P_{c}, T_{c}, F_{c}\right)$, the $W_{c}$ activities are compacted based on the following rules :

1. Rule $_{1}$

$$
\begin{aligned}
& \exists t_{i}, t_{j} \in \mathrm{T}, p_{i j} \in \mathrm{P} \text { where } \\
& \mathrm{P}=\left\{p_{1}, \ldots, p_{i j}, \ldots, p_{n}\right\} \\
& \mathrm{P}^{\prime}=\mathrm{P}-\left\{p_{i j}\right\} \\
& \mathrm{T}=\left\{t_{1}, \ldots, t_{i}, t_{j}, \ldots, t_{m}\right\} \\
& \mathrm{T}^{\prime}=\mathrm{T}-\left\{t_{i}\right\} \\
& \mathrm{F}=\left\{f_{1}, \ldots,\left(t_{i}, p_{i j}\right),\left(p_{i j}, t_{j}\right), \ldots, f_{k}\right\} \\
& \mathrm{F}^{\prime}=\mathrm{F}-\left(\left\{\left(t_{i}, p_{i j}\right),\left(p_{i j}, t_{j}\right)\right\} \cup\left\{\left(p_{k}, t_{i}\right) / p_{k} \in \bullet t_{i}\right\}\right) \cup\left\{\left(p_{k}, t_{j}\right) / p_{k} \in \bullet t_{i}\right\} \\
& \mathrm{C}=\operatorname{seq}\left(t_{i}, t_{j}, p_{i j}\right), t_{i} \in T_{i n t} \text { and } t_{j} \in T_{i n t}
\end{aligned}
$$




$$
\text { Rule }_{1}: \frac{(P, T, F), C}{\left(P^{\prime}, T^{\prime}, F^{\prime}\right)}
$$

This rule shows that if we dispose of an internal activity $t_{i}$ followed by another internal activity $t_{j}$ and linked to it via $p_{i j}$, then we eliminate $t_{i}$ and $p_{i j}$ as well as all the flows between $t_{i}$ and $t_{j}$. Moreover, we get rid of all the flows coming from $\bullet t_{i}$ to $t_{i}$ and create new flows linking the places belonging to $\bullet t_{i}$ to $t_{j}$. This case is illustrated in figure 18 .

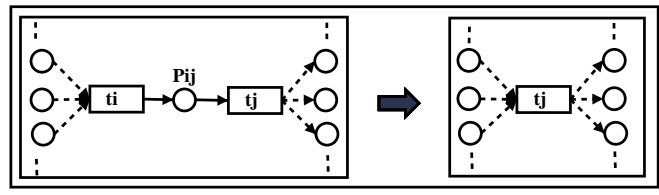

Fig. 18. Rule $_{1}$ : Compaction of two sequent internal activities

In our example, $t_{1}$ and $t_{2}$ are sequent activities and both of them is internal. By applying rule $_{1}$ we eliminate the activity $t_{1}$, the place $p_{1}$ and the flows $\left(t_{1}, p_{1}\right)$ and $\left(p_{1}, t_{2}\right)$ that link $t_{1}$ to $t_{2}$ and create the new flow $\left(i, t_{2}\right)$ that links the source i to $t_{2}$ (figure 19).

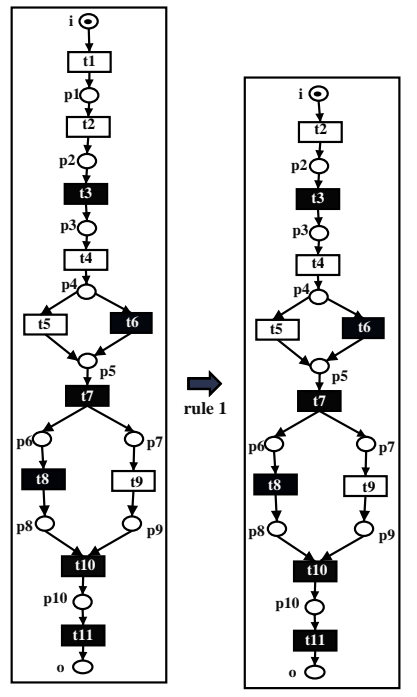

Fig. 19. result of rule $_{1}$ application 
2. Rule $_{2}$

$$
\begin{aligned}
& \exists t_{i}, t_{j} \in \mathrm{T}, p_{i j} \in \mathrm{P} \text { where } \\
& \mathrm{P}=\left\{p_{1}, \ldots, p_{i j}, \ldots, p_{n}\right\} \\
& \mathrm{P} '=\mathrm{P}-\left\{p_{i} j\right\} \\
& \mathrm{T}=\left\{t_{1}, \ldots, t_{i}, t_{j}, \ldots, t_{m}\right\} \\
& \mathrm{T}^{\prime}=\mathrm{T}-\left\{t_{i}\right\} \\
& \mathrm{F}=\left\{f_{1}, \ldots,\left(t_{i}, p_{i j}\right),\left(p_{i j}, t_{j}\right), \ldots, f_{k}\right\} \\
& \mathrm{F}^{\prime}=\mathrm{F}-\left(\left\{\left(t_{i}, p_{i j}\right),\left(p_{i j}, t_{j}\right)\right\} \cup\left\{\left(p_{k}, t_{i}\right) / p_{k} \in \bullet t_{i}\right\}\right) \cup\left\{\left(p_{k}, t_{j}\right) / p_{k} \in \bullet t_{i}\right\} \\
& \mathrm{C}=\operatorname{seq}\left(t_{i}, t_{j}, p_{i j}\right), t_{i} \in T_{\text {int }} \text { and } t_{j} \in T_{\text {coop }} \\
& \qquad \text { Rule }_{2}: \frac{(P, T, F), C}{\left(P^{\prime}, T^{\prime}, F^{\prime}\right)}
\end{aligned}
$$

Rule $_{2}$ shows that if we dispose of an internal activity $t_{i}$ followed by a cooperative activity $t_{j}$ and linked to it via $p_{i j}$, then we eliminate $t_{i}, p_{i j}$ and all the flows existing between $t_{i}$ and $t_{j}$ (ie $\left(t_{i}, p_{i j}\right)$ and $\left(p_{i j}, t_{j}\right)$ ). In addition, we eliminate all the flows coming from $\bullet t_{i}$ to $t_{i}$ and create new ones linking the places belonging to $\bullet t_{i}$ to $t_{j}$. This case is illustrated in figure 20 .

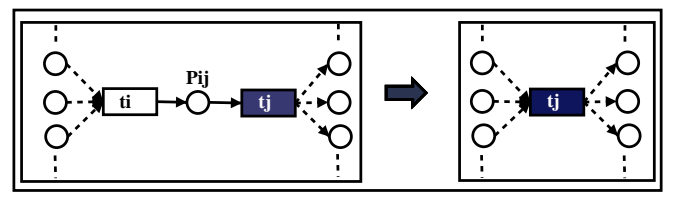

Fig. 20. Rule $_{2}$ : Compaction of an internal activity followed by a cooperative activity

In figure $21, t_{2}$ is an internal activity followed by a cooperative activity $t_{3}$ and linked to it via $p_{2}$, then rule $_{2}$ can be applied and as a result we eliminate the activity $t_{2}$, the place $p_{2}$ and the flows $\left(\mathrm{i}, t_{2}\right),\left(t_{2}, p_{2}\right)$ and $\left(p_{2}, t_{3}\right)$ and create a new flow linking the source i to $t_{3}$ (figure 21 ).

3. Rule $_{3}$

$$
\begin{aligned}
& \exists t_{i}, t_{j} \in \mathrm{T}, p_{i j} \in \mathrm{P} \text { where } \\
& \mathrm{P}=\left\{p_{1}, \ldots, p_{i j}, \ldots, p_{n}\right\} \\
& \mathrm{P}^{\prime}=\mathrm{P}-\left\{p_{i j}\right\} \\
& \mathrm{T}=\left\{t_{1}, \ldots, t_{i}, t_{j}, \ldots, t_{m}\right\} \\
& \mathrm{T}^{\prime}=\mathrm{T}-\left\{t_{j}\right\} \\
& \mathrm{F}=\left\{f_{1}, \ldots,\left(t_{i}, p_{i j}\right),\left(p_{i j}, t_{j}\right), \ldots, f_{k}\right\} \\
& \mathrm{F}^{\prime}=\mathrm{F}-\left(\left\{\left(t_{i}, p_{i j}\right),\left(p_{i j}, t_{j}\right)\right\} \cup\left\{\left(t_{j}, p_{k}\right) / p_{k} \in t_{j} \bullet\right\}\right) \cup\left\{\left(t_{i}, p_{k}\right) / p_{k} \in t_{j} \bullet\right\} \\
& \mathrm{C}=\operatorname{seq}\left(t_{i}, t_{j}, p_{i j}\right), t_{i} \in T_{\text {coop }} \text { and } t_{j} \in T_{i n t} \\
& \qquad \text { Rule }_{3}: \frac{(P, T, F), C}{\left(P^{\prime}, T^{\prime}, F^{\prime}\right)}
\end{aligned}
$$

Here we show another case of sequent activities compaction where we dispose of a cooperative activity $t_{i}$ followed by an internal one $t_{j}$ and linked to it 


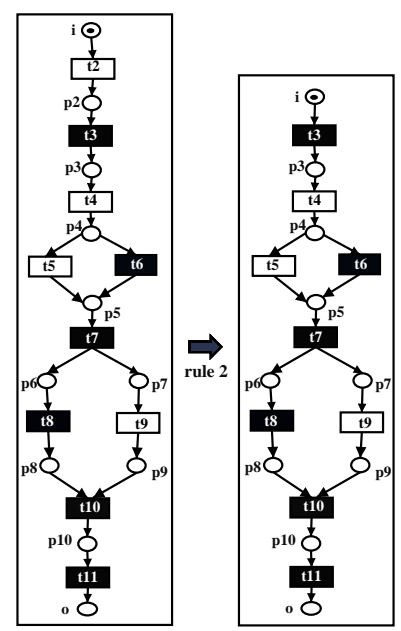

Fig. 21. result of rule $_{2}$ application

via $p_{i j}$. In this case, we remove the internal activity $t_{i}$, the place $p_{i j}$ and all the flows belonging to $\bullet t_{j}$. Besides, we create new flows linking $t_{i}$ to all the places belonging to $t_{j} \bullet$. This scenario is illustrated in figure 22 .

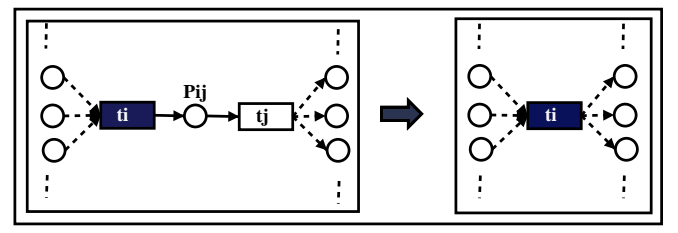

Fig. 22. Rule $_{3}$ : Compaction of a cooperative activity with an internal activity

The application of rule $_{3}$ to $t_{2}, t_{3}$ of figure 21 results in removing the internal activity $t_{4}$, the place $p_{3}$ as well as the flows $\left(t_{3}, p_{3}\right),\left(p_{3}, t_{4}\right)$ and $\left(t_{4}, p_{4}\right)$, and creating the new flow that relies $t_{3}$ to $p_{4}$ (figure 23 ).

4. Rule $_{4}$

$\exists t_{i} \in T_{i n t}, t_{j} \in \mathrm{T}, p_{i j}^{i}, p_{i j}^{o} \in \mathrm{P}$ where

$\mathrm{T}=\left\{t_{1}, \ldots, t_{i}, t_{j}, \ldots, t_{m}\right\}$

$\mathrm{T}^{\prime}=\mathrm{T}-\left\{t_{i}\right\}$

$\mathrm{F}=\left\{f_{1}, \ldots,\left(p_{i j}^{i}, t_{i}\right),\left(t_{i}, p_{i j}^{o}\right), \ldots, f_{k}\right\}$

$\mathrm{F}^{\prime}=\mathrm{F}-\left\{\left(p_{i j}^{i}, t_{i}\right),\left(t_{i}, p_{i j}^{o}\right)\right\}$

$\mathrm{C}=\operatorname{alt}\left(t_{i}, t_{j}, p_{i j}^{i}, p_{i j}^{o}\right)$ and $t_{i} \in T_{\text {int }}$ 


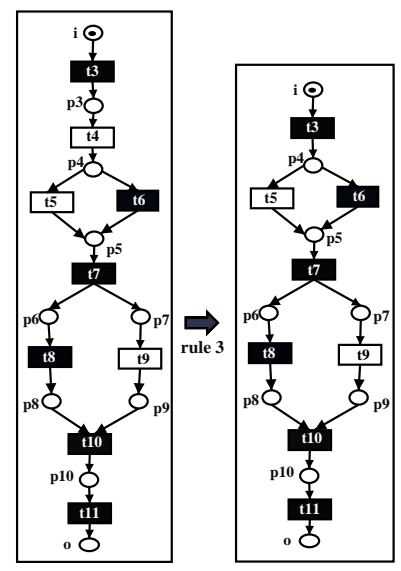

Fig. 23. result of rule $_{3}$ application

$$
\text { Rule }_{4}: \frac{(P, T, F), C}{\left(P, T^{\prime}, F^{\prime}\right)}
$$

In this rule we show that if we dispose of two alternative activities $t_{i}$ and $t_{j}$ where $t_{i}$ is internal and $t_{j}$ can either be internal or cooperative, then we remove $t_{i}$ as well as all the flows that are directly linked to it. This case is illustrated in figure 24 .
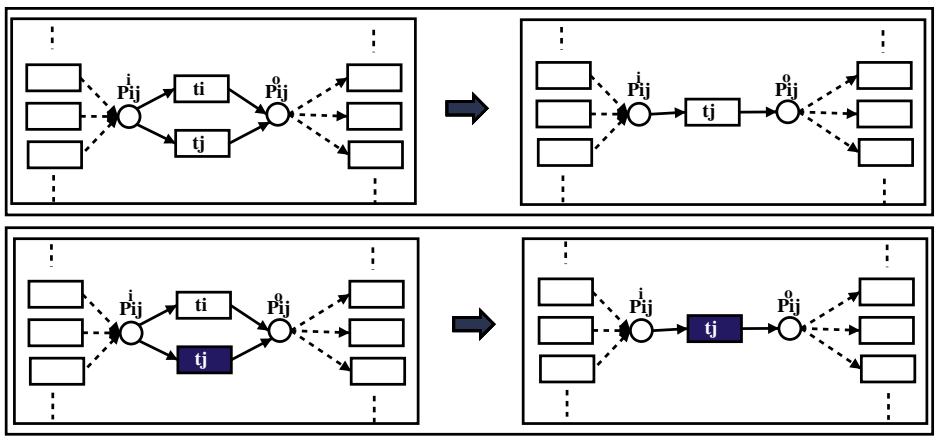

Fig. 24. Rule $_{4}$ : Compacting alternative activities

Rule $_{4}$ can be applied to the activities $t_{5}$ and $t_{6}$ of figure 23 that fulfills all its conditions and as a result we eliminate $t_{5}$ and the flows $\left(p_{4}, t_{5}\right)$ and $\left(t_{5}\right.$, $\left.p_{5}\right)$. The obtained workflow is shown in figure 25 . 


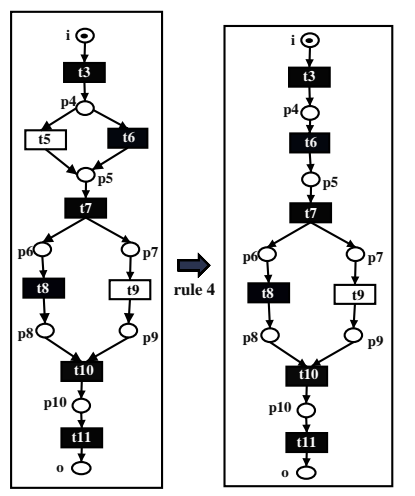

Fig. 25. result of rule $_{4}$ application

5. Rule $_{5}$

$\exists t_{i}, t_{j} \in \mathrm{T}, p_{k i}, p_{i l}, p_{k j}, p_{j l} \in \mathrm{P}$ where

$\mathrm{P}=\left\{p_{1}, \ldots, p_{k i}, p_{i l}, \ldots, p_{n}\right\}$

$\mathrm{P}^{\prime}=\mathrm{P}-\left\{p_{k i}, p_{i l}\right\}$

$\mathrm{T}=\left\{t_{1}, \ldots, t_{i}, t_{j}, \ldots, t_{m}\right\}$

$\mathrm{T}^{\prime}=\mathrm{T}-\left\{t_{i}\right\}$

$\mathrm{F}=\left\{f_{1}, \ldots,\left(t_{k}, p_{k i}\right),\left(p_{k i}, t_{i}\right),\left(t_{i}, p_{i l}\right),\left(p_{i l}, t_{l}\right), \ldots, f_{k}\right\}$

$\mathrm{F}^{\prime}=\mathrm{F}-\left\{\left(t_{k}, p_{k i}\right),\left(p_{k i}, t_{i}\right),\left(t_{i}, p_{i l}\right),\left(p_{i l}, t_{l}\right)\right\}$

$\mathrm{C}=\operatorname{sync}\left(t_{i}, t_{j}, p_{k i}, p_{i l}, p_{k j}, p_{j l}\right)$ and $t_{i} \in T_{i n t}$

$$
\text { Rule }_{5}: \frac{(P, T, F), C}{\left(P^{\prime}, T^{\prime}, F^{\prime}\right)}
$$

The objective of this rule is to remove internal activities in a synchronized flow. Hence, if an internal activity $t_{i}$ is synchronized with an activity $t_{j}$ that can be either internal or cooperative, then $t_{i}$ as well as all the places and flows directly linked to it are removed. This case is illutrated in figure 26 .

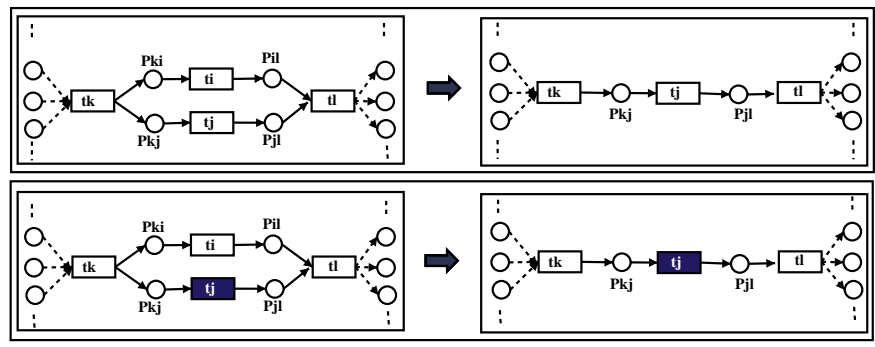

Fig. 26. Rule $_{5}$ : Compacting parallel activities 
In our example, the activities $t_{8}$ and $t_{9}$ of figure 25 fullfills all the conditions of this rule and applying rule $_{5}$ results in removing the internal activity $t_{9}$, the places $p_{7}$ and $p_{9}$, and the flows $\left(t_{7}, p_{7}\right),\left(p_{7}, t_{9}\right),\left(t_{9}, p_{9}\right)$ and $\left(p_{9}, t_{1} 0\right)$. The obtained workflow is illutrated in figure 27. Finally, we notice that none of the five rules could be applied any more. As a consequence, the compacting procedure is stopped and the resulting workflow (figure 27) is the cooperative workflow corresponding to the internal workflow of figure 17.

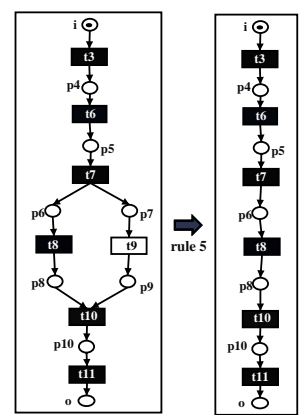

Fig. 27. result of rule $_{5}$ application

The resut of the five rules' application on the minimal connected workflow of figure 17 is summarized in Figure 28.

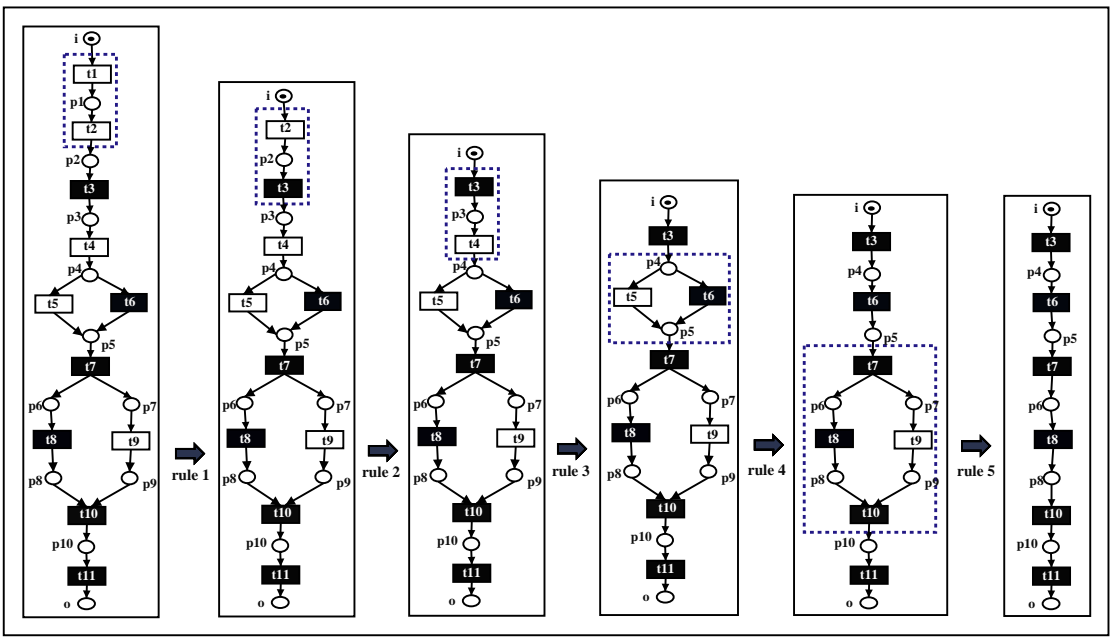

Fig. 28. Workflow Compacting 